\title{
A DENSITY-ASSISTED PARTICLE FILTER ALGORITHM FOR TARGET TRACKING WITH UNKNOWN BALLISTIC COEFFICIENT
}

\author{
Marcelo G. S. Bruno and Anton Pavlov \\ Instituto Tecnológico de Aeronáutica \\ São José dos Campos SP 12228-900, Brazil \\ ph:(55-12) 3947-5878; email: \{bruno,anton\} @ele.ita.br
}

\begin{abstract}
We present in this paper a density-assisted particle filter (DAPF) algorithm for ballistic target tracking with unknown, fixed ballistic coefficient. The proposed algorithm uses an optimized importance function to update the particle population and then utilizes the updated particles and their respective importance weights to build a parametric approximation of the joint posterior probability density function (pdf) of the target state and the unknown ballistic coefficient. A new set of particles is then resampled according to this approximate pdf and propagated to the next iteration of the algorithm. Simulation results confirm previous claims in the literature that DAPFs are viable alternatives for sequential estimation in nonlinear dynamic models with unknown, static parameters.
\end{abstract}

\section{INTRODUCTION}

Ballistic target tracking using conventional radar measurements is a difficult problem due to inherent nonlinearities in both the target motion model and the radar observation model that preclude the direct application of optimal linear sequential estimators such as the Kalman filter. As an alternative to linearized Kalman-type filters, Farina et al proposed in [1] a sequential Monte Carlo solution to the problem of ballistic target tracking during re-entry into the atmosphere using a standard sampling/importance resampling (SIR) particle filter [2]. More recently [3], an improved SIR ballistic target tracking filter was proposed combining an optimized measurement-based importance function [4] and Metropolis-Hastings [5] move steps. References [1] and [3] assumed, however, that the target ballistic coefficient was deterministic and perfectly known to the tracking filter, which may not be realistic in practical situations. In this paper, we consider the alternative scenario where the ballistic coefficient is fixed (time-invariant), but is also random and unknown.

Efficient sequential state estimation in nonlinear dynamic models with unknown, static parameters is still an open problem in particle filtering theory. As pointed out in [6], the

The work of the second author was supported by CAPES, Brazil. conventional solution of extending the state vector to include the unknown parameter vector and then applying standard SIR filtering is bound to fail with static parameters due to the lack of ergodicity of the extended state process. A possible solution, see [6], is to assume an artificial dynamic evolution for the unknown parameter vector. However, such solution has the disadvantage of treating the fixed parameters as time-variant when, in reality, they are not. Recently, Djurić et al [7] suggested the use of density-assisted particle filters (DAPFs) as a potentially viable alternative to perform joint sequential state and parameter estimation without explicitly introducing an artificial dynamic model for the static parameters. In this paper, we design a modified sequential Monte Carlo filter that combines the optimized importance function from [3] and the density-assisted particle filtering technique from [7]. Next, we investigate the performance of the proposed DAPF in a realistic problem of target tracking with an unknown (random), fixed ballistic coefficient.

The paper is divided into five sections. Section 1 is this Introduction. In Section 2, we review the target motion and observation models for the ballistic target tracking problem. In Section 3, we detail the proposed DAPF tracking algorithm. In Section 4, we present simulation results and compare the DAPF tracker to the alternative modified artificial parameter evolution method in [8]. Finally, we summarize the conclusions of our work in Section 5.

\section{MOTION AND OBSERVATION MODEL}

Let $k$ be a non-negative integer number and denote by $\Delta$ the time interval between two consecutive radar measurements. Let also superscript $T$ denote the transpose of a vector or matrix. Assuming for simplicity a flat Earth, we define the target state at instant $t=k \Delta$ as the four-dimensional vector

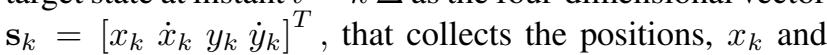
$y_{k}$, and the velocities, $\dot{x}_{k}$ and $\dot{y}_{k}$, of the target in a system of $2 \mathrm{D}$ cartesian coordinates $(x, y)$. We also define the twodimensional observation vector $\mathbf{z}_{k}=\left[\begin{array}{ll}r_{k} & \varepsilon_{k}\end{array}\right]^{T}$, that collects the measured range, $r_{k}$, and elevation angle, $\varepsilon_{k}$, of the target at instant $t=k \Delta$. We model the random sequences $\left\{\mathbf{s}_{k}\right\}$, 
$k \geq 0$, and $\left\{\mathbf{z}_{k}\right\}, k \geq 1$, by the nonlinear state-space model $[1,3]$

$$
\begin{aligned}
\mathbf{s}_{k+1} & =\mathbf{\Psi}\left(\mathbf{s}_{k}, \beta\right)+\mathbf{w}_{k} \\
\mathbf{z}_{k} & =\mathbf{h}\left(\mathbf{s}_{k}\right)+\mathbf{v}_{k}
\end{aligned}
$$

where $\beta$ is the target ballistic coefficient, assumed timeinvariant, unknown and random with uniform distribution in $\left[\beta_{L}, \beta_{U}\right]$. On the other hand, $\left\{\mathbf{w}_{k}\right\}_{k \geq 0}$ and $\left\{\mathbf{v}_{k}\right\}_{k \geq 1}$ are assumed to be two mutually independent, identically distributed (i.i.d.) Gaussian random sequences, also independent of $\beta$ and $\mathbf{s}_{0}$, and with zero mean and non-singular covariance matrices respectively

$$
\mathbf{Q}=q\left[\begin{array}{cc}
\boldsymbol{\Theta} & \mathbf{0} \\
\mathbf{0} & \boldsymbol{\Theta}
\end{array}\right], \quad \boldsymbol{\Theta}=\left[\begin{array}{cc}
\frac{\Delta^{3}}{3} & \frac{\Delta^{2}}{2} \\
\frac{\Delta^{2}}{2} & \Delta
\end{array}\right]
$$

and

$$
\mathbf{R}=\left[\begin{array}{cc}
\sigma_{r}^{2} & 0 \\
0 & \sigma_{\varepsilon}^{2}
\end{array}\right]
$$

where $q, \sigma_{r}^{2}$ and $\sigma_{\varepsilon}^{2}$ are positive real numbers. The nonlinear function $\boldsymbol{\Psi}($.$) in (1) is in turn given by$

$$
\mathbf{\Psi}\left(\mathbf{s}_{k}, \beta\right)=\mathbf{\Phi} \mathbf{s}_{k}+\mathbf{G} \mathbf{f}\left(\mathbf{s}_{k}, \beta\right)+\mathbf{G}\left[\begin{array}{c}
0 \\
-g
\end{array}\right]
$$

where $g$ denotes the gravity acceleration (assumed constant and known), matrices $\Phi$ and $\mathbf{G}$ are given by

$$
\boldsymbol{\Phi}=\left[\begin{array}{cccc}
1 & \Delta & 0 & 0 \\
0 & 1 & 0 & 0 \\
0 & 0 & 1 & \Delta \\
0 & 0 & 0 & 1
\end{array}\right], \quad \mathbf{G}=\left[\begin{array}{cc}
\frac{\Delta^{2}}{2} & 0 \\
\Delta & 0 \\
0 & \frac{\Delta^{2}}{2} \\
0 & \Delta
\end{array}\right]
$$

and $\mathbf{f}\left(\mathbf{s}_{k}, \beta\right)$ denotes the aerodynamic drag force whose expression is [1]

$$
\mathbf{f}\left(\mathbf{s}_{k}\right)=-0.5 \frac{g}{\beta} \rho\left(y_{k}\right) \sqrt{\dot{x}_{k}^{2}+\dot{y}_{k}^{2}}\left[\begin{array}{l}
\dot{x}_{k} \\
\dot{y}_{k}
\end{array}\right] .
$$

In (7), $\rho($.$) is the air density which decays with the alti-$ tude $y_{k}$ according to the exponential law described in $[1,3]$. Finally, assuming that the true target elevation angle lies between 0 and $\pi / 2$, the nonlinear function $\mathbf{h}($.$) in (2) is writ-$ ten as

$$
\mathbf{h}\left(\mathbf{s}_{k}\right)=\left[\begin{array}{c}
\sqrt{x_{k}^{2}+y_{k}^{2}} \\
\arctan \left(\frac{y_{k}}{x_{k}}\right)
\end{array}\right] .
$$

\section{DENSITY-ASSISTED PARTICLE FILTER TRACKER}

Let $\mathbf{z}_{1: k}=\left\{\mathbf{z}_{1}, \mathbf{z}_{2}, \ldots, \mathbf{z}_{k}\right\}$ be a sequence of observations. A particle-filter strategy for joint sequential state and parameter estimation is to generate in a recursive fashion a set of samples $\left\{\left(\mathbf{s}_{k}^{(j)}, \beta_{k}^{(j)}\right), j=1, \ldots, N_{p}\right\}, k \geq 0$, with associated weights $\left\{w_{k}^{(j)}\right\}$, such that the weighted average $\sum_{j=1}^{N_{p}} w_{k}^{(j)}\left[\mathbf{s}_{k}^{(j)} \beta_{k}^{(j)}\right]^{T}$ converges (in some statistical sense) at each instant $k$ to the joint MMSE state and parameter estimate $\mathbb{E}\left\{\left[\mathbf{s}_{k} \beta\right]^{T} \mid \mathbf{z}_{1: k}\right\}$.

Let $q\left(\mathbf{s}_{k} \mid \mathbf{s}_{0: k-1}, \beta_{0: k-1}, \mathbf{z}_{1: k}\right)$ be a chosen proposal probability density function (pdf), referred to as the importance function. The density-assisted particle filter (DAPF) tracker derived in this paper, see also [7], is a modified sampling/importance resampling (SIR) [2] that builds an $a p$ proximate properly weighted set to represent $p\left(\mathbf{s}_{k}, \beta \mid \mathbf{z}_{1: k}\right)$ using the following recursive algorithm:

1. For $j=1, \ldots, N_{p}$, sample $\mathbf{s}_{0}^{(j)} \sim p\left(\mathbf{s}_{0}\right)$ and $\beta_{0}^{(j)} \sim$ $p(\beta)$.

2. Set $\mathrm{k}=1$.

3. For $j=1, \ldots, N_{p}$

- Draw $\tilde{\mathbf{s}}_{k}^{(j)} \sim q\left(\mathbf{s}_{k} \mid \mathbf{s}_{0: k-1}^{(j)}, \beta_{0: k-1}^{(j)}, \mathbf{z}_{1: k}\right)$, and make $\tilde{\beta}_{k}^{(j)}=\beta_{k-1}^{(j)}$.

- Update the weights

$$
\tilde{w}_{k}^{(j)} \propto \frac{p\left(\mathbf{z}_{k} \mid \tilde{\mathbf{s}}_{k}^{(j)}\right) p\left(\tilde{\mathbf{s}}_{k}^{(j)} \mid \mathbf{s}_{k-1}^{(j)}, \beta_{k-1}^{(j)}\right)}{q\left(\tilde{\mathbf{s}}_{k}^{(j)} \mid \mathbf{s}_{0: k-1}^{(j)}, \beta_{0: k-1}^{(j)}, \mathbf{z}_{1: k}\right)} .
$$

where the symbol $\propto$ denotes "proportional to" and $\sum_{j} \tilde{w}_{k}^{(j)}=1$.

End-for

4. Build a parametric approximation $\widehat{p}\left(\mathbf{s}_{k}, \beta \mid \mathbf{z}_{1: k}\right)$ to the true posterior pdf $p\left(\mathbf{s}_{k}, \beta \mid \mathbf{z}_{1: k}\right)$ using the particle set $\left\{\left(\tilde{\mathbf{s}}_{k}^{(j)}, \tilde{\beta}_{k}^{(j)}\right)\right\}$ and the respective weights $\left\{\tilde{w}_{k}^{(j)}\right\}$.

5. For $j=1, \ldots, N_{p}$, resample $\left(\mathbf{s}_{k}^{(j)}, \beta_{k}^{(j)}\right) \sim \widehat{p}\left(\mathbf{s}_{k}, \beta \mid\right.$ $\left.z_{1: k}\right)$.

6. Make $k=k+1$ and go back to step 3 .

The DAPF tracker above differs from conventional SIR filtering applied to the extended state vector $\left[\mathbf{s}_{k} \beta\right]^{T}$ in the sense that we replace standard resampling with replacement according to the particle weights by resampling according to the approximate parametric pdf $\widehat{p}\left(\mathbf{s}_{k}, \beta \mid \mathbf{z}_{1: k}\right)$. As long as the parametric approximation of the true posterior is reasonably accurate, the previously described algorithm implies a natural evolution in time of the sample set $\left\{\beta_{k}^{(j)}\right\}$ without the severe depletion of particles that would otherwise occur, see [7], if standard resampling according to the weights were used. 


\subsection{Importance Function Design}

In order to reduce particle degeneracy [2] arising from the increase over time in the variance of the particle weights, one should choose the optimal importance function

$$
q\left(\mathbf{s}_{k} \mid \mathbf{s}_{0: k-1}^{(j)}, \beta_{0: k-1}^{(j)}\right)=p\left(\mathbf{s}_{k} \mid \mathbf{s}_{k-1}^{(j)}, \beta_{k-1}^{(j)}, \mathbf{z}_{k}\right)
$$

that minimizes [4] the variance of $\tilde{w}_{k}^{(j)}$ conditioned on the simulated particle trajectories and on the observations. However, a closed-form analytical expression for the optimal importance function cannot be obtained in this particular application due to the nonlinearities in both the state model in (1) and the observation model in (2). Using a technique proposed in [4], we expand then equation (2) around $\boldsymbol{\Psi}\left(\mathbf{s}_{k-1}^{(j)}, \beta_{k-1}^{(j)}\right)$ and build the first-order linear approximation

$$
\begin{aligned}
\mathbf{z}_{k} & \approx \mathbf{h}\left[\mathbf{\Psi}\left(\mathbf{s}_{k-1}^{(j)}, \beta_{k-1}^{(j)}\right)\right]+\mathbf{H}_{k}^{(j)}\left[\mathbf{s}_{k}-\mathbf{\Psi}\left(\mathbf{s}_{k-1}^{(j)}, \beta_{k-1}^{(j)}\right)\right] \\
& +\mathbf{v}_{k},
\end{aligned}
$$

where $\mathbf{H}_{k}^{(j)}=\nabla \mathbf{h}(\mathbf{s})$ evaluated at $\mathbf{s}=\mathbf{\Psi}\left(\mathbf{s}_{k-1}^{(j)}, \beta_{k-1}^{(j)}\right)$, and

$$
\nabla \mathbf{h}(\mathbf{s})=\left[\begin{array}{cccc}
\frac{s[1]}{\sqrt{s^{2}[1]+s^{2}[3]}} & 0 & \frac{s[3]}{\sqrt{s^{2}[1]+s^{2}[3]}} & 0 \\
\frac{-s[3]}{s^{2}[1]+s^{2}[3]} & 0 & \frac{s[1]}{s^{2}[1]+s^{2}[3]} & 0
\end{array}\right] .
$$

Let $\mathcal{N}(\mathbf{s}-\mathbf{a}, \mathbf{P})$ denote the multivariable normal function of argument $\mathbf{s}$, mean $\mathbf{a}$ and covariance matrix $\mathbf{P}$. Assuming the linearized observation model in (11), it is possible to show that $p\left(\mathbf{s}_{k} \mid \mathbf{s}_{k-1}^{(j)}, \beta_{k-1}^{(j)}, \mathbf{z}_{k}\right)$ may be approximated by $\mathcal{N}\left(\mathbf{s}_{k}-\mathbf{m}_{k}^{(j)}, \boldsymbol{\Sigma}_{k}^{(j)}\right)$ where

$$
\begin{aligned}
\boldsymbol{\Sigma}_{k}^{(j)} & =\left[\mathbf{Q}^{-1}+\left(\mathbf{H}_{k}^{(j)}\right)^{T} \mathbf{R}^{-1}\left(\mathbf{H}_{k}^{(j)}\right)\right]^{-1} \\
\mathbf{m}_{k}^{(j)} & =\left(\Sigma_{k}^{(j)}\right)\left\{\mathbf{Q}^{-1} \mathbf{\Psi}\left(\mathbf{s}_{k-1}^{(j)}, \beta_{k-1}^{(j)}\right)\right. \\
& +\left(\mathbf{H}_{k}^{(j)}\right)^{T} \mathbf{R}^{-1}\left[\mathbf{z}_{k}-\mathbf{h}\left(\mathbf{\Psi}\left(\mathbf{s}_{k-1}^{(j)}, \beta_{k-1}^{(j)}\right)\right)\right. \\
& \left.\left.+\mathbf{H}_{k}^{(j)} \mathbf{\Psi}\left(\mathbf{s}_{k-1}^{(j)}, \beta_{k-1}^{(j)}\right)\right]\right\}
\end{aligned}
$$

\subsection{Parametric Approximation of the Posterior PDF}

For simplicity, we follow the suggestion in [7] and use the parametric approximation

$$
\widehat{p}\left(\mathbf{s}_{k}, \beta \mid \mathbf{z}_{1: k}\right) \approx \mathcal{N}\left(\mathbf{s}_{k}-\underline{\mu}_{k \mid \beta}, \mathbf{P}_{k \mid \beta}\right) \mathcal{B}\left(\beta ; \lambda_{1, k}, \lambda_{2, k}\right)
$$

where $\mathcal{B}\left(\beta ; \lambda_{1, k}, \lambda_{2, k}\right)$ denotes the shifted Beta pdf defined in the interval $\left[\beta_{L}, \beta_{U}\right]$ with shape parameters $\lambda_{1, k}$ and $\lambda_{2, k}$. Let $\underline{\tilde{\alpha}}_{k}^{(j)}=\left[\left(\begin{array}{ll}\left.\tilde{\mathbf{s}}_{k}^{(j)}\right)^{T} & \tilde{\beta}_{k}^{(j)}\end{array}\right]^{T}, j=1, \ldots, N_{p}\right.$, and compute at instant $k$ the weighted sample mean, $\underline{\mu}_{k}$, and the weighted sample covariance, $\mathbf{P}_{k}$, given by

$$
\begin{aligned}
\underline{\mu}_{k} & =\sum_{j=1}^{N_{p}} \tilde{w}_{k}^{(j)} \underline{\tilde{\alpha}}_{k}^{(j)}=\left[\left(\underline{\mu}_{s, k}\right)^{T} \mu_{\beta, k}\right]^{T} \\
\mathbf{P}_{k} & =\sum_{j=1}^{N_{p}} \tilde{w}_{k}^{(j)}\left(\underline{\tilde{\alpha}}_{k}^{(j)}-\underline{\mu}_{k}\right)\left(\underline{\tilde{\alpha}}_{k}^{(j)}-\underline{\mu}_{k}\right)^{T} \\
& =\left[\begin{array}{cc}
\mathbf{P}_{s s, k} & \mathbf{P}_{s \beta, k} \\
\mathbf{P}_{s \beta, k}^{T} & \sigma_{\beta, k}^{2}
\end{array}\right] .
\end{aligned}
$$

For $N_{p}$ sufficiently large, we can estimate the parameters $\lambda_{1, k}$ and $\lambda_{2, k}$ directly from $\mu_{\beta, k}$ and $\sigma_{\beta, k}^{2}$ by solving the implicit equations (adapted from [7])

$$
\begin{aligned}
\frac{\mu_{\beta, k}-\beta_{L}}{\left(\beta_{U}-\beta_{L}\right)} & =\frac{\lambda_{1, k}}{\lambda_{1, k}+\lambda_{2, k}} \\
\frac{\sigma_{\beta, k}^{2}}{\left(\beta_{U}-\beta_{L}\right)^{2}} & =\frac{\lambda_{1, k} \lambda_{2, k}}{\left(\lambda_{1, k}+\lambda_{2, k}\right)^{2}\left(\lambda_{1, k}+\lambda_{2, k}+1\right)}
\end{aligned}
$$

In the sequel, we make the parameter vector $\underline{\mu}_{k \mid \beta}$ equal to the linear least squares estimate of $\mathbf{s}_{k}$ given $\beta$ and $\mathbf{z}_{1: k}$, i.e.,

$$
\underline{\mu}_{k \mid \beta}=\underline{\mu}_{s, k}+\mathbf{P}_{s \beta, k} \sigma_{\beta, k}^{-2}\left(\beta-\mu_{\beta, k}\right) .
$$

The corresponding conditional covariance matrix is then

$$
\mathbf{P}_{k \mid \beta}=\mathbf{P}_{s s, k}-\mathbf{P}_{s \beta, k}\left(\sigma_{\beta, k}^{-2}\right) \mathbf{P}_{s \beta, k}^{T} .
$$

\section{SIMULATION RESULTS}

We simulated the state model in (1) with parameters $g=$ $9.8 \mathrm{~m} / \mathrm{s}^{2}, q=5, \Delta=2 \mathrm{~s}, \beta_{L}=35000 \mathrm{~kg} \cdot \mathrm{m}^{-1} \cdot \mathrm{s}^{-2}$, and $\beta_{U}=45000 \mathrm{~kg} \cdot \mathrm{m}^{-1} \cdot \mathrm{s}^{-2}$. The observation model in (2) was simulated assuming $\sigma_{r}=75 \mathrm{~m}$, and $\sigma_{\epsilon}=0.017$ rad. The initial state $\mathbf{s}_{0}$ was specified as a Gaussian random vector with mean $\mathbf{m}_{0}=\left[232000 \mathrm{~m} 2290 \cos \left(190^{\circ}\right) \mathrm{m} / \mathrm{s}\right.$ $\left.88000 \mathrm{~m} 2290 \sin \left(190^{\circ}\right) \mathrm{m} / \mathrm{s}\right]$ and diagonal covariance matrix $\Sigma_{0}$ with $\Sigma_{0}(1,1)=\Sigma_{0}(3,3)=1000^{2} m^{2}$, and $\Sigma_{0}(2,2)=$ $\Sigma_{0}(4,4)=20^{2} m^{2} \cdot s^{-2}$. The simulated target is tracked over 50 time steps using the DAPF tracker described in Section 3 with $N_{p}=6000$ particles.

For comparison purposes, we also implemented the stateof-the-art joint state/parameter estimator proposed by Liu and West in [8]. The filter in [8] is a modified auxiliary particle filter (APF) [9] that assumes an artificial evolution for the static model parameters which may in turn be reinterpreted as equivalent to a kernel smoothing method with shrinkage, see [8] for details. Figures 1(a) and (b) show the root-mean square error (RMSE) curves for the target position estimates obtained by the DAPF and the Liu-West filter (LWF) respectively in the $x$ and $y$ coordinates. The RMSE curves were estimated from 100 independent Monte Carlo runs. As a global reference for performance, we also plot in 


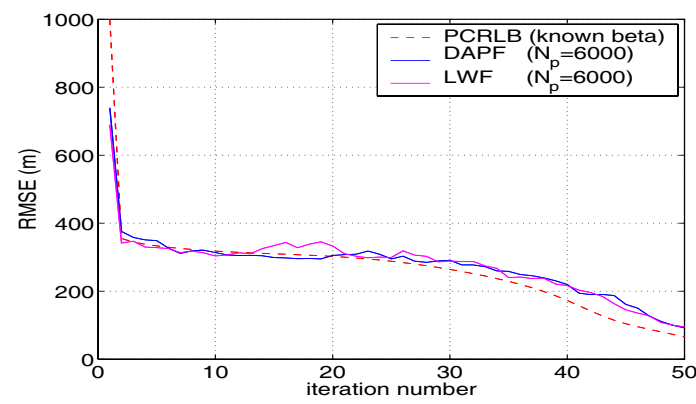

(a)

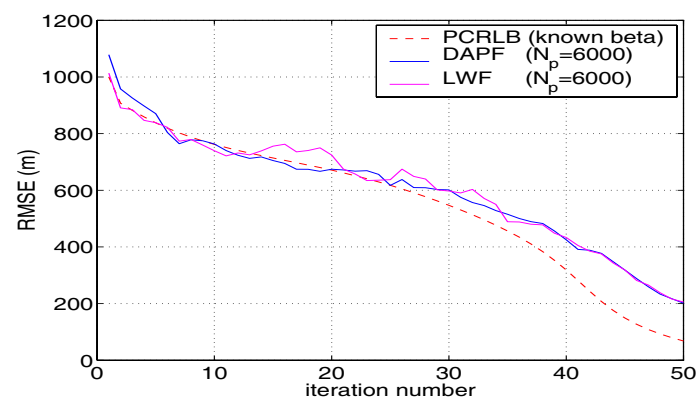

(b)

Fig. 1. RMS position estimation error for the DAPF tracker and the Liu-West filter with $\sigma_{r}=75 \mathrm{~m}$ and $\sigma_{\dot{x}_{0}}=\sigma_{\dot{y}_{0}}=$ $20 \mathrm{~m} / \mathrm{s}$; (a) $x$ coordinate, (b) $y$ coordinate.

Figures 1(a) and (b) the square-root of the posterior CramérRao lower bound (PCRLB) that would have been achieved by the ideal (optimal) nonlinear filter if the ballistic coefficient were perfectly known. The PCRLB was computed using a Monte Carlo approximation of the recursive algorithm in [10]. The curves in Figure 1 show that, despite the use of a rough parametric approximation to the true posterior pdf, the performance of the proposed DAPF tracker is identical within the margin of error of the simulations to the performance of the alternative Liu-West filter.

\section{CONCLUSIONS}

We presented in this paper a modified sequential Monte Carlo filter for target tracking with unknown, fixed ballistic coefficient. The proposed filter jointly estimates the hidden dynamic target state and the unknown, static ballistic coefficient combining the density-assisted particle filtering technique in [7] with a locally optimized measurement-based importance function. Empirical evidence from our simulation results seem to confirm the claim in [7] that densityassisted particle filters (DAPFs) are a potentially viable alternative for combined sequential estimation of dynamic states and static parameters. Future work will include a detailed theoretical analysis of the convergence properties of DAPFs and the derivation of optimal parametric approximations to the true posterior pdf to ensure good convergence behavior.

\section{REFERENCES}

[1] A. Farina, B. Ristic, and D. Benvenuti, "Tracking a ballistic target: comparison of several nonlinear filters," IEEE Transactions on Aerospace and Electronic Systems, vol. 38, n. 3, pp.854-867, July 2002.

[2] M.S. Arulampalam, S. Maskell, N. Gordon, and T. Clapp, "A tutorial on particle filters for online nonlinear/non-Gaussian Bayesian tracking," IEEE Transactions on Signal Processing, vol. 50, n. 2, pp.174-188, February 2002.

[3] M. G. S. Bruno and A. Pavlov, "Improved Particle Filters for Ballistic Target Tracking," Proceedings IEEE ICASSP 2004, vol. II, pp. 705-708, Montreal, Quebec, Canada, May 17-21, 2004.

[4] A. Doucet, S. J. Godsill, and C. Andrieu,"On sequential Monte Carlo sampling methods for Bayesian filtering," Stat. Comput., vol.10, pp. 197-208, 2000.

[5] W. R. Gilks and C. Berzuini,"Following a moving target - Monte Carlo inference for dynamic Bayesian models," J. R. Statist. Soc. B, vol.63, pp. 127-146, 2001.

[6] C. Andrieu, A. Doucet, S. S. Singh, and V. B. Tadić, "Particle Methods for Change Detection, System Identification, and Control," Proceedings of the IEEE, vol.92, n.3, pp. 423-438, March 2004.

[7] P. M. Djurić, M. F. Bugallo, and J. Míguez, "Density assisted particle filters for state and parameter estimation," Proceedings IEEE ICASSP 2004, vol.II, pp. 701-704, Montreal, Quebec, Canada, May 17-21, 2004.

[8] J. Liu and M. West, "Combined parameter and state estimation in simulation-based filtering," in Sequential Monte Carlo Methods in Practice, A. Doucet, J. F. G. Freitas, and N. J. Gordon, Editors, pp.197-223, New York: Springer-Verlag, 2001.

[9] M. K. Pitt and N. Shephard, "Filtering via simulation: Auxiliary particle filters," Journal of the American Statistical Association, 94(446), pp. 590-599, 1999.

[10] P. Tichavský, C. H. Muravchik, and A. Nehorai, "Posterior Cramér-Rao Bounds for Discrete-Time Nonlinear Filtering," IEEE Transactions on Signal Processing, vol.46, n.5, May 1998. 